\title{
Evaluation of Different Pretreatment Methods for Xylooligosaccharides Production
}

\author{
Dolmani Amat ${ }^{1,2 *}$ and Livleen Shukla ${ }^{1}$ \\ ${ }^{1}$ ICAR-Indian Agricultural Research Institute, Division of Microbiology, New Delhi, India \\ ${ }^{2}$ ICAR-Indian Institute of Soil Science, Nabibagh, Berasia Road, Bhopal, India \\ *Corresponding author
}

\section{A B S T R A C T}

\begin{tabular}{|l|}
\hline Ke y w or d s \\
Xylooligo- \\
saccharides, \\
Corncob, \\
Pretreatment \\
\hline Article Info \\
\hline $\begin{array}{l}\text { Accepted: } \\
\text { 08 December } 2020 \\
\text { Available Online: } \\
\text { 10 January } 2021\end{array}$ \\
\hline
\end{tabular}

Xylooligosaccharides are emerging group of prebiotics that exert beneficial effects on human beings and other animals when supplemented with their diets as these are not digested in the upper gastrointestinal tract and help in proliferation of beneficial microbes. In the present study corn cobs were pretreated with dilute acid, dilute alkali and steam, for the production of xylooligosaccharides. The pretreatment methods were evaluated based on removal of lignin after the pretreatment and release of reducing sugars after saccharification with commercial xylanase. It was observed that, alkali pretreatment method was found to be best pretreatment method as it could remove maximum amount of lignin $(5.7 \%)$. Also, alkali pretreated corncobs could able to release maximum amount $(71.7 \pm 1.1 \mathrm{mg}$ per $\mathrm{g}$ of substrate) of reducing sugar upon saccharification than steam and acid pretreated corncobs. Hundred miligrams of xylobiose was obtained per $\mathrm{g}$ of alkali pretreated corncob (on dry weight basis) after $24 \mathrm{hr}$ using partially purified xylanase. Thus the present study proves the suitability of alkali pretreatment for the production of xylooligosaccahride from corn cob.

\section{Introduction}

Corncobs are important by-product of the corn industry that is used either as animal feed or returned to the harvested fields (Aachary and Prapulla, 2009) for composting. In India nearly 9.3 million hectares of area was under maize cultivation with the production of about 28.64 million tons in 2019-20 (Government of India, 2020) from which approximately 4.98 million metric tons of corncobs could be generated. Corn cobs have many industrial applications like production of bioethanol (Chen et al., 2007), biochar (Mullen et al., 2000), for decontamination of water (Billon et al., 2006) etc. Corn cobs are rich in xylan up to $35 \%$ that can be used for food applications like production of xylitol, xylose, xylooligosaccharides (XOs) and xylanases (Aachary and Prapulla, 2009).

Xylooligosaccharides (XOs) are a class of non-digestible food ingredient that beneficially affects the host by selectively stimulating the growth and/or activity of one 
or a limited number of bacteria in the colon, and thus improves the host health (Moure et al., 2006). XOs are oligomers containing 2-10 xylose units linked by $\beta-1,4$ xylosidic linkage.

Among the different XOs, xylobioses are important for food applications. Thus, production of XOs from corn cobs will not only solve the problem of proper disposal of these wastes but also generate income and improve the nutritional status of the mass of people.

Xylooligosaccharides can be produced from corn cob by autohydrolysis, chemical, enzymatic and chemo enzymatic methods. XOs production using autohydrolysis lead to undesirable products like lignin, furfurals and monosaccharides (Zhu et al., 2006a) which requires further purifications.

In chemical methods, both acids and alkalis are known to produce excess of xylose and toxic products i.e. furfural and hydroxymethyl furfural (HMF) at elevated temperatures which are detrimental for food applications (Yang et al., 2005). However, chemo enzymatic method involves mild use of chemical that break the xylan- lignin linkage thus making the xylanase accessible to the xylan for the production of xylooligosaccharides without any toxic byproducts. Hence, it is most widely used method for the production of xylooligosaccharides.

Different chemo-enzymatic method using i.e. aqueous ammonia (Zhu et al., 2006b), dilute acids, alkali and sodium hypochlorite (Gowdhaman and Ponnusami, 2015) have been carried out for xylooligosaccharides production.

In the present study, different pretreatment methods were evaluated for xylooligosaccharides production.

\section{Materials and Methods}

\section{Procurement and processing of substrate}

Dried corncobs were procured from Indian Institute of Maize Research, Pusa campus, New Delhi. Dried corncobs were milled in a hammer mill at Division of Agricultural Engineering, IARI. These milled corncobs were passed through $500 \mu \mathrm{m}$ brass sieve to get a uniform size (Plate 1).

\section{Pretreatment of substrate}

\section{Alkali pretreatment}

Alkali pretreatment of corncob was carried out by modified method of Ai et al., (2005) where powdered corncobs were treated with $2 \% \mathrm{NaOH}$ solution at solid to liquid ratio of 1:6 (w/w) and kept for 6h. After 6h, pretreated corncobs were washed with deionized water to bring down the $\mathrm{pH}$ to 6.0 and then dried in an oven at $40^{\circ} \mathrm{C}$ and substrate was kept in an air tight container till further use.

\section{Acid pretreatment}

Acid pretreatment of corn cob was carried out by method described by Yang et al (2005) with some modification. Briefly, corncob powder was soaked in $0.1 \%$ (w/v) $\mathrm{H}_{2} \mathrm{SO}_{4}$ solution in a ratio of $1: 10(\mathrm{w} / \mathrm{v})$ for $6 \mathrm{~h}$ at $60^{\circ} \mathrm{C}$. The excess acid was removed using deionized water till the $\mathrm{pH}$ of the washings was 6.0 and pretreated corncob powder was dried at $40^{\circ} \mathrm{C}$ and kept in an air tight container till further use.

\section{Steam pretreatment}

Steam pretreatment was carried out using a mini steamer, followed by washing with deionized water till the $\mathrm{pH}$ of the washings was 6.0 and pretreated corncob powder was 
dried at $40^{\circ} \mathrm{C}$ and kept in an air tight container till further use.

\section{Evaluation of pretreatment methods}

Pretreatment methods were evaluated based on content of xylan and lignin in the corncob after pretreatment (Plate 2) and release of reducing sugars (xylose equivalent) after saccharification of pretreated corncobs with commercial xylanase.

\section{Analysis of structural carbohydrate of corncob}

In both raw and pretreated corn cobs, xylan content was determined by TAPPI method (1997), cellulose content by Updegroff (1969), lignin and ash content by NREL (Sluiter et al., 2012) method. Pretreatment method which preserved maximum content of xylan and removed maximum lignin were selected.

\section{Saccharification of pretreated corncobs}

After selecting the pretreatment methods based on xylan and lignin content, the pretreated substrates were further subjected to saccharification with commercial xylanase.Pretreated substrate releasing maximum reducing sugars (xylose equivalent) upon saccharification was considered as best pretreatment method for xylooligosaccharides production. Enzymatic hydrolysis of pretreated substrates was carried out according to NREL-LAP method (Selig et al., 2008) with modification. Briefly $0.1 \mathrm{~g}$ of pretreated corncob was taken in a $50-\mathrm{ml}$ screw capped plastic bottle. To this commercial xylanase $(25,000 \mathrm{IU} / \mathrm{g}$ from SRL Pvt. Ltd) was added @ 600IU/g of dry substrate. Volume was made to $10 \mathrm{ml}$ using citrate buffer $\mathrm{pH} 4.8$ and then incubated at $50^{\circ} \mathrm{C}$ in shaking water bath for $24 \mathrm{~h}$ at $150 \mathrm{rpm}$. Aliquot was taken after $24 \mathrm{~h}$ and was centrifuged at 5000rpm for $10 \mathrm{~min}$. The supernatant was used for estimation of reducing sugar by DNSA method (Miller, 1959).

\section{Production of xylooligosaccharide from corncob}

Production of xylooligosaccharides was carried out by the method described by Aachary and Prapulla (2009) with some modification. In a screw capped bottle, $0.1 \mathrm{~g}$ alkali pretreated corncob was taken. To the bottle, partially purified endoxylanase from Aspergillus fumigatus SKF-4 was added @ 6 $\mathrm{IU} / \mathrm{ml}$ and volume was made up to $10 \mathrm{ml}$ with $0.05 \mathrm{M}$ citrate buffer $\mathrm{pH} 4.8$.

\section{Results and Discussion}

\section{Evaluation of various economically feasible pre-treatment methods}

After pretreatment of corn cobs using alkali $(2 \% \mathrm{NaOH})$, acid $\left(0.1 \% \mathrm{H}_{2} \mathrm{SO}_{4}\right)$ and steam, these substrates along with raw corncob were subjected to compositional analysis. Table 1 shows the xylan and lignin content of raw and pretreated corncobs. Based on the results, it has been observed that raw corncobs had 34.7 $\pm 1.5 \%$ xylan and $16.5 \pm 2.0 \%$, lignin. Among the pretreatments, steam pretreated corncobs had maximum xylan $(30.1 \pm 4.5 \%)$ and lignin $(14.8 \pm 2.8 \%)$ content. It has also been observed that alkali pretreatment although showed lower xylan content $(20.5 \pm 0.2 \%)$ as compared to steam but owing to lower content of lignin $(10.8 \pm 1.3 \%)$ would help in further enzymatic hydrolysis for xylooligosaccharides (XOs) production. Acid pretreated corncobs showed lowest xylan content $(16.3 \pm 0.8 \%)$ which clearly shows the non-feasibility of this pretreatment for XOs production and it also had $13.1 \pm 3.2 \%$ lignin. 
Structural analysis of alkali pretreated corn cobs

After selecting the alkali pretreatment as the best for XOs production, the changes in content $(\% \mathrm{w} / \mathrm{w})$ of cellulose, ash, and other components along with xylan and lignin in corncobs was determined after alkali pretreatment. Fig 1 show that after alkali pretreatment, xylan content decreased from $34.7 \pm 1.5 \%$ to $29.5 \pm 0.2 \%$ and lignin from $16.5 \pm 2.0 \%$ to $11.0 \pm 1.3 \%$ also ash content decreased in alkali pretreated corncobs from $1.6 \pm 0.3 \%$ to $0.4 \pm 0.1 \%$. But after the pretreatment there was enrichment of cellulose concentration to $56.0 \pm 2.3 \%$ in alkali pretreated corncobs as compared to $41.5 \pm 5.0 \%$ in raw corncobs. Others components of corncobs which were not determined in this study were calculated by deducting total weight of the xylan, lignin, cellulose and ash from 100. Others components denotes pectins, proteins and the extractives (soluble non-structural material such as non-structural sugars, nitrogenous material, chlorophyll and waxes).

Evaluation of pretreatment method based on enzymatic hydrolysis of pretreated corncobs

Based on xylan and lignin content, steam and alkali pretreated corncobs were found to be suitable for enzymatic hydrolysis. For exploring further the feasibility of acid pretreatment for xylooligosaccharides (XOs) production, all the pretreated corncobs along with the raw corncobs were used for enzymatic hydrolysis using commercial xylanase (25000 IU/g from SRL Pvt. Ltd.) @ 600 IU per $g$ of dry substrate. The assumption is that pretreated substrate releasing maximum reducing sugars would be considered as the best pretreatment for XOs production. Fig 2 shows that among the pretreated corncobs, alkali pretreated corncobs showed maximum release of reducing sugars (mg per $\mathrm{g}$ of dry substrate) i.e. $71.7 \pm 1.1$ followed by steam pretreated corncobs $(50.4 \pm 0.8)$ and the last acid pretreated corncobs $(34.3 \pm 1.5)$. The lowest level of reducing sugars was released from raw corncobs. Results clearly indicates the need of pretreatment for the production of XOs and alkali pretreatment method was taken as the best pretreatment method for XOs production.

\section{Production of xylooligosaccharide from alkali pretreated corncobs}

Upon enzymatic hydrolysis of alkali pretreated corncobs using $1 \%(\mathrm{w} / \mathrm{v})$ substrate loading and $6 \mathrm{IU} / \mathrm{ml}$ partially purified xylanase from Aspergillus fumigatus SKF-4, $100 \mathrm{mg}$ of xylobiose was produced per $\mathrm{g}$ of alkali pretreated corncob on dry weight basis after $24 \mathrm{~h}$. Because among the xylooligosaccarides, xylobiose is important for food application, concentration of xylobiose in the hydrolysate was given more importance.

Table.1 Xylan and lignin content in raw and pretreated corncobs

\begin{tabular}{|c|c|c|}
\hline Corncobs & $\begin{array}{c}\text { Xylan } \\
(\boldsymbol{\%} \mathbf{w} / \mathbf{w})\end{array}$ & $\begin{array}{c}\text { Lignin } \\
(\boldsymbol{\%} \mathbf{w} / \mathbf{v})\end{array}$ \\
\hline Raw & $34.7 \pm 1.5$ & $16.5 \pm 2.0$ \\
\hline Alkali pretreated & $29.5 \pm 0.2$ & $10.8 \pm 1.3$ \\
\hline Acid pretreated & $16.3 \pm 0.8$ & $13.1 \pm 3.2$ \\
\hline Steam pretreated & $30.1 \pm 4.5$ & $14.8 \pm 2.8$ \\
\hline
\end{tabular}


Fig.1 Compositional analysis of a) raw corncob and b) alkali pretreated corncobs

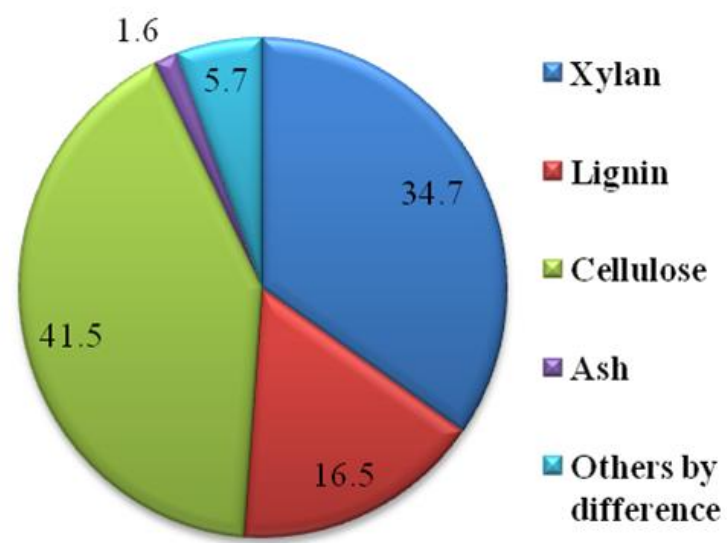

b)

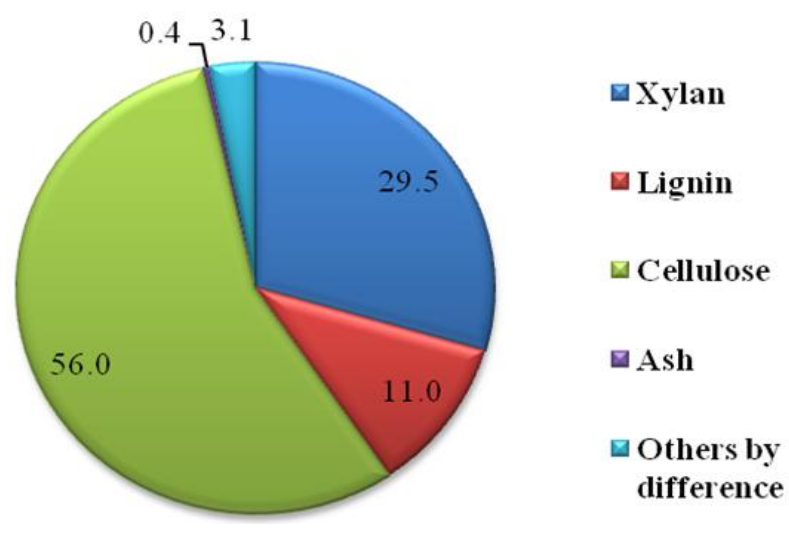

Fig.2 Reducing sugars released from raw and pretreated corncobs after treatment with commercial xylanase

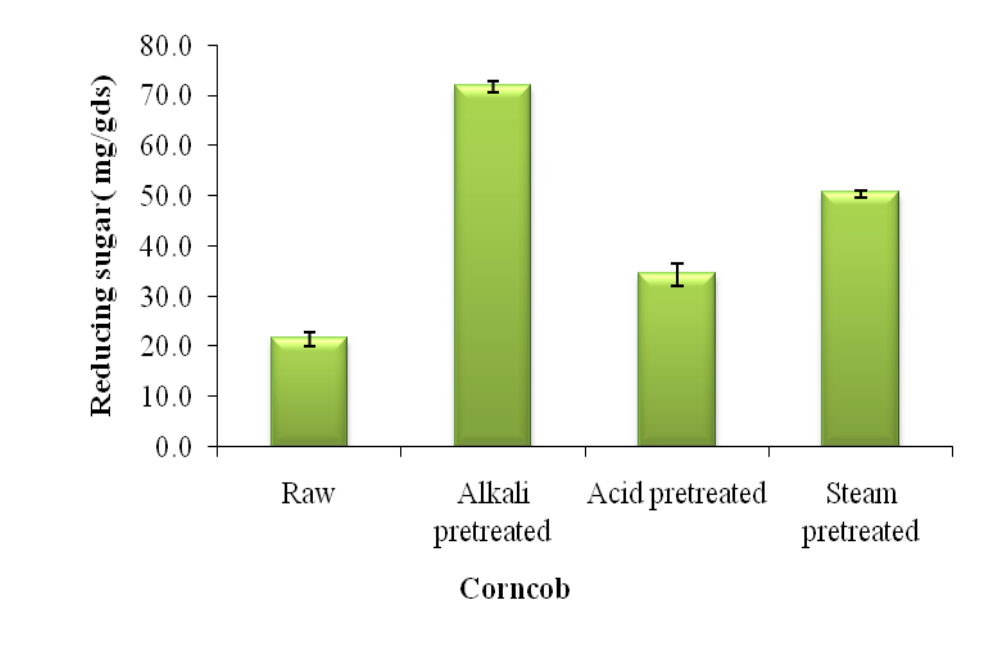


Plate.1 Procurement and processing of corncobs. (A) Dried corncobs procured from IIMR, New Delhi, (B) milling of corncobs at Division of Agricultural Engineering, (C) sieving of milled corncobs, (D) sieved corncobs

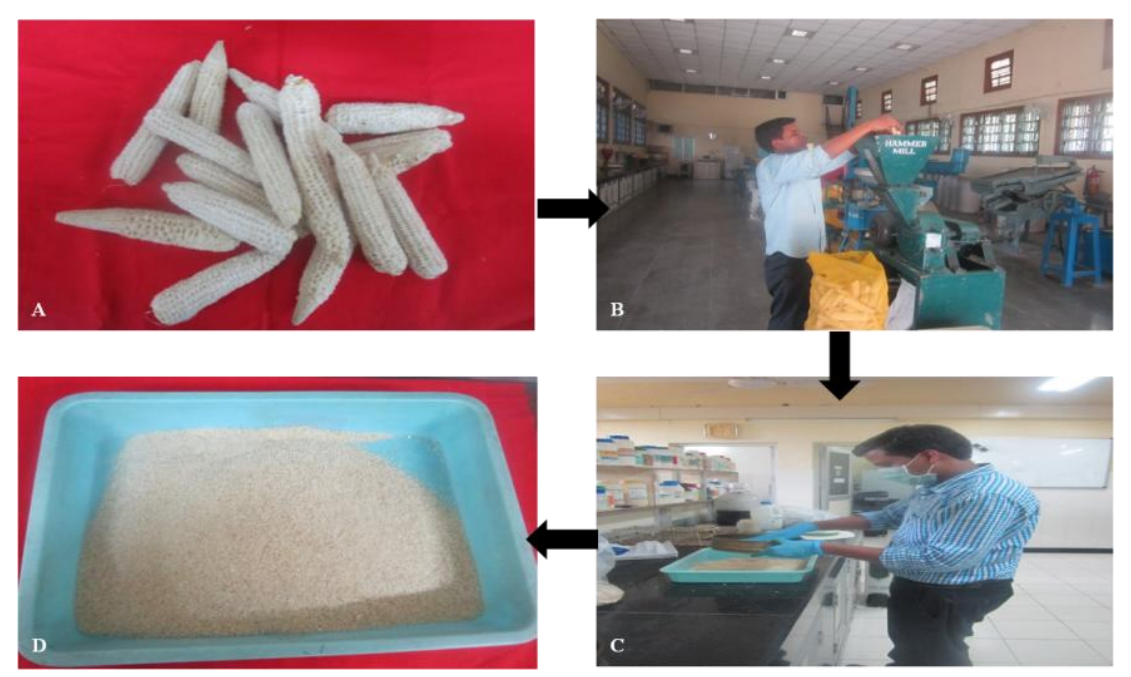

Plate.2 Raw and pretreated corncobs

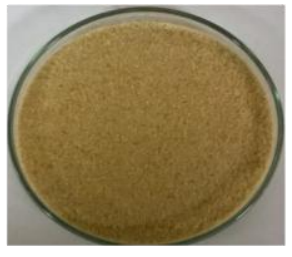

Acid pretreated corn cobs

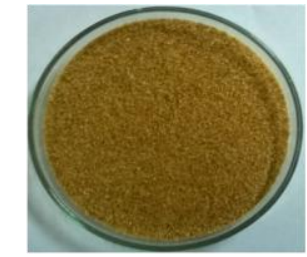

Alkali pretreated corncobs

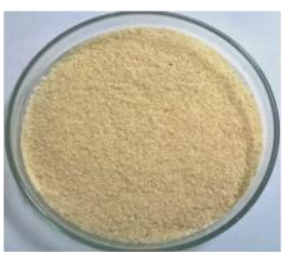

Raw corncobs

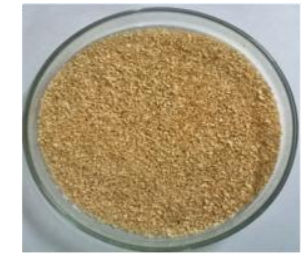

Steam pretreated corncobs

\section{Evaluation of different pretreatment methods}

Corncobs as a source of lignocellulosic material composed of cellulose, hemicellulose and lignin, minute quantity of protein pectins, extractives which comprises non-structural sugars, chlorophyll, nitrogenous material, and waxes) and ash. Accessibility of xylan component of cell wall matrix for enzymatic reaction is restricted by the surrounding lignin network as well as ester and ether lignin- carbohydrate linkages. Before enzymatic hydrolysis xylan-lignin bonds need to be broken. The process of making xylan available for enzymatic reaction without the complete extraction is known as pretreatment. By the pretreatment methods, lignin is either broken down or removed from the biomass. There are several pretreatment methods like mechanical, chemicals, thermo-chemicals, biological pretreatments have been widely used for lignocellulosic biomass (Sun and Cheng, 2002) but chemical methods are most 
effective and practiced for industrial applications. Pretreatment of corncobs was carried out using steam, acid and alkali by following standard protocols. After pretreatment, content of hemicellulose (xylan) and lignin in the raw and pretreated substrates were evaluated (Table 1). Based on the results, it has been observed that raw corncobs contained $34.7 \pm 1.5 \%$ xylan and $16.5 \pm 2.0 \%$ lignin (on dry weight basis). Reports on the chemical composition of corncobs, one of the most important LCMs studied for xylooligosaccharides (XOs) production, suggest the presence of high xylan content (30- 35\%) (Tan et al., 2008). Similar results were also reported where 34.8 $\mathrm{g}$ of xylan per $100 \mathrm{~g}$ of raw corncobs which is at par with the present study (Yang et al., 2005). Lignin content of $17.0 \%$ (Eylen et al., 2011), 17.7\% (Garrote et al., 2002) have been reported in raw corncobs.. Among the pretreatments, steam pretreated corncobs had maximum xylan $(30.1 \pm 4.5 \%)$ and lignin $(14.8 \pm 2.8 \%)$ content. Steam pretreated corncobs had highest content of xylan because in steam pretreatment there is partial hydrolysis of hemicellulose and alteration of lignin structure than the alkali and acid pretreatment. Steam pretreatment cause loosening of biomass and increase the accessible surface area (Mosier et al., 2005). Xylan and lignin content of the steam pretreated corncobs was lower than that of raw corncobs indicating their removal during washing with deionized water after pretreatment. During steam pretreatment there is disruption of structural component of biomass due to high temperature and pressure. Depending upon the severity of applied conditions, steam pretreatment results in significant depolymerization of lignin component and its solublization during subsequent extraction (Kumar et al., 2011). It has also been observed that alkali pretreatment although showed lower xylan content $(20.5 \pm 0.2 \%)$ as compared to steam but it also had lowest content of lignin (10.8 \pm $1.3 \%$ ) among the pretreated corncobs which would help in further enzymatic hydrolysis process for XOs production. Alkali pretreatment involves alteration in the structure of lignin, partial decrystalization of cellulose and partial solvation of hemicellulose (Ibrahim et al., 2011) thus lowering their content in the pretreated corncobs. Acid pretreated corncobs showed lowest xylan content $(16.3 \pm 0.8 \%)$ which clearly shows the non feasibility of this pretreatment for XOs production and it also had $13.1 \pm 3.2 \%$ lignin.It is because of degradation of xylan in the acid pretreated corncob to xylose under acidic pretreatment. Hemicellulose and lignin get solubilised during acid pretreatments and when concentrated acid is used, even cellulose gets hydrolysed (Sun and Cheng, 2002). Xylan content of acid pretreated corncobs was lowest (39.2\%) among the pretreated corncobs (Aachary and Prapulla, 2009).

Comparatively higher content of lignin was observed in acid pretreated corncobs $(13.1 \pm 3.2 \%)$ than the alkali pretreatment $(10.8$ $\pm 1.3 \%$ ). This result coincides with the findings, where lesser APPL (acid precipitated lignin) was recovered from acid pretreated Parthenium sp $(1.0 \pm 0.0 \mathrm{mg} / \mathrm{g})$ than the alkali pretreated substrates $(7.53 \pm$ $0.5 \mathrm{mg} / \mathrm{g}$ ) (Pandiyan et al., 2013). Though there is removal of lignin due to dilute acid pretreatment still dilute acids are less effective in removing lignin compared to alkaline methods (Bensah and Mensah, 2013).

\section{Changes in structural components of corncobs after alkali pretreatment}

After selecting the alkali pretreatment as the best for XOs production, the changes in content $(\% \mathrm{w} / \mathrm{w})$ of cellulose, ash, and other components along with xylan and lignin in corncobs was determined after alkali 
pretreatment. Fig. 1 shows that after alkali pretreatment, xylan content decreased from $34.7 \pm 1.5 \%$ to $29.5 \pm 0.2 \%$ and lignin from $16.5 \pm 2.0 \%$ to $11.0 \pm 1.3 \%$. After alkali pretreatment, there was decrease in the xylan and lignin content in the pretreated corncobs than the raw one because the alkaline solution caused swelling, leading to an increase in the internal surface area, a reduction in the degree of polymerization (DP) and crystallinity, also disruption of the crystalline structure by separation of structural linkages between lignin, hemicellulose, and cellulose (Brodeur et al., 2011). Hence there is release of lignin and hemicellulose from the pretreated corncobs resulting in decrease in their content in the pretreated substrate. Ash content decreased in alkali pretreated corncobs from $1.6 \pm 0.3 \%$ to $0.4 \pm 0.1 \%$. Ash is composed of inorganic materials that are bound in the physical structure of the biomass. Boonchuay et al (2014) also reported reduction in ash content from $1.37 \pm 0.03 \%$ in raw corncobs to $0.47 \pm 0.03 \%$ in alkali pretreated corncobs, which coincides with the results obtained during research. But after the pretreatment there was enrichment of cellulose to $56.0 \pm$ $2.3 \%$ in alkali pretreated corncobs as compared to $41.5 \pm 5.0 \%$ in raw corncobs. Similar results have been reported earlier also where after alkali pretreatment, cellulose content of corncobs increased from $36 \%$ in raw corncobs to $50 \%$ in alkali pretreated corncobs (Sahare et al., 2012). Decrease in the content of lignin and hemicellulose in the pretreated substrates has resulted in enrichment of cellulose content in the pretreated corncobs than the raw substrate which shows that larger polymers have been broken down to smaller one. Reduction in the content of other components (pectins, proteins and the extractives) in alkali pretreated corncobs might be due to release of some of the components which are soluble in water during washing with deionized water after pretreatment.
Enzymatic hydrolysis of corncobs for selection of efficient pretreatment method

After determination of xylan and lignin content of the pretreated corncobs, they were used for enzymatic hydrolysis using commercial xylanase for further evaluation of pretreatment methods.. Fig. 2 shows that among the pretreated corncobs, alkali pretreated corncobs showed maximum release of reducing sugars (mg per $\mathrm{g}$ of dry substrate) i.e. $71.7 \pm 1.1$, followed by steam pretreated corncobs (50.4 \pm 0.8$)$ and the last acid pretreated corncobs $(34.3 \pm 1.5)$. Results indicate that pretreatment of corncobs is needed for improving the enzymatic hydrolysis as reducing sugars released from pretreated corncobs was more than the raw corncobs. Similar results were reported where more reducing sugars were released by alkali pretreated Parthenium sp than the acid pretreated one upon saccharification with Accelerase 1500 (Pandiyan et al., 2013). Alkali treatment is considered to be a promising pretreatment method which alters the structural properties such as accessible surface area and crystallinity of pretreated substrate thereby enhancing its enzymatic hydrolysis (Wang et al., 2010). Pandiyan et al (2013) also reported that release of reducing sugars from the pretreated substrate is inversely proportional to its lignin content. Hence, as in case of steam pretreatment, as there is less removal of lignin than alkali pretreatment, it has resulted in low amount of release of reducing sugars due to hindrance of lignin for the accessibility of enzyme. But acid pretreated corncobs resulted in release of lowest amount of reducing sugars due to the degradation of xylan during the pretreatment process thus reducing the amount of substrate available for the xylanase enzyme for hydrolysis. Based on above results, alkali pretreatment proves to be suitable pretreatment of corncobs and was finally selected for production of 
xylooligosaccharides. Similarly, Aachary and Prapulla (2009) also found that among different pretreatment methods screened for xylooligosaccharides production, alkali pretreated corncobs was found to be best for xylooligosaccharides production. Alkaline process requires less severe conditions compared with the other pretreatment methods, also it requires less energy and is less expensive. Alkali pretreatments can be performed at room temperature (Alvira et al., 2010) and causes minimal sugar loss and reported to be more effective on agricultural residues than woody materials (Kumar et al., 2009). Pre-treatment of LCMs with alkali is likely to be the most efficient and safest for xylooligosaccharides production (Jain et al., 2015). Further optimization with respect to concentration of alkali $(\mathrm{NaOH})$, biomass to alkali ratio, residence time is required to improve the pretreatment efficiency.

\section{Production of xylooligosaccharides from alkali pretreated corncobs}

After the preliminary study confirmed the suitability of the purified endoxylanase from Aspergillus fumigatus SKF-4 for production of xylooligosaccharides using beechwood xylan, then alkali pretreated corncobs was used for production of xylobiose using partially purified endoxylanase from Aspergillus fumigatus SKF-4 because xylan from beechwood as a substrate for commercial production of xylobiose are expensive due to its high cost. Hence, corncobs as a cheap source of xylan were taken as alternative substrate. Results showed that upon enzymatic hydrolysis for $24 \mathrm{~h}, 100$ $\mathrm{mg}$ of xylobiose per $\mathrm{g}$ of alkali pretreated corncob was produced in the enzymatic hydrolysate indicating the need of optimizing the process parameters affecting the enzymatic hydrolysis of xylan for enhanced production of xylobiose. Several researchers have reported the production of xylooligosaccharides from alkali pretreated corn cobs. Boonchuay (2014) carried out production of xylooligosaccharides from alkali pretreated corncob using crude thermostable endo-xylanase (100U/g) from Streptomyces Thermovulgaris TISTR1948 and xylobiose obtained was $85.15 \pm 1.18 \mathrm{mg} / \mathrm{g}$ of substrate after 12 hour. Thus, in the present study alkali pretreatment was found to be most efficient pretreatment method and was suitable for xylooligosaccharides production from corn cobs.

\section{Acknowledgements}

The authors are thankful to Post Graduate School, ICAR-Indian Agricultural Research Institute (IARI) and Indian Council of Agricultural Research (ICAR) for providing financial help in conducting the research work.

\section{References}

Aachary, A. A. and Prapulla, S. G. 2009. Value addition to corncob: production and characterization of xylooligosaccharides from alkali pretreated lignin-saccharide complex using Aspergillus oryzae MTCC 5154. Bioresour.Technol.,100, 991-995

Government of India. 2020. First Advance Estimates of Production of Food grains for 2020-21, Retrieved Oct 14, 2020 from

http://agricoop.nic.in/sites/default/files/F irstEstimate2020-21.pdf.

Chen, M., Xia, L.and Xue, P. 2007. Enzymatic hydrolysis of corncob and ethanol production from cellulosic hydrolysate. Int. Biodeterior Biodegradation, 59(2), 85-89.

Mullen, C. A., Boateng, A. A., Goldberg, N. M., Lima, I. M., Laird, D. A. and Hicks, K. B. 2000. Bio-oil and bio-char production from corn cobs and stover by 
fast pyrolysis. Biomass Bioenergy, 34(1), 67-74.

Billon, L., Meric, V., Castetbon, A., Francois, J. and Desbrieres, J. 2006. Removal of copper ions from water of broilers by a modified natural based, corncobs. J. Appl. Poly. Sci., 102, 4637-4645

Moure, A., Gullon, P., Dominguez, H. and Parajo, J.C. 2006. Advances in the manufacture, purification and applications of xylo-oligosaccharides as food additives and nutraceuticals. Process Biochem., 41, 1913 - 1923.

Zhu, S., Wu, Y., Yu, Z., Zhang, X., Li, H. and Gao, M.2006a. The effect of microwave irradiation on enzymatic hydrolysis of rice straw. Bioresour.Technol. 97, 1964 $-1968$.

Yang, R., Xu, S., Wang, Z. and Yang, W. 2005. Aqueous extraction of corncob xylan and production of xylooligosaccharides. LWT-Food Sci. Technol., 38, 677 - 682

Zhu, Y., Kim, T.H., Lee, Y.Y., Chen, R. and Elander, R.T.2006b. Enzymatic production of xylooligosaccharides from corn stover and corn cobs treated with aqueous ammonia. In Twenty-Seventh Symposium on Biotechnology for Fuels and Chemicals (pp. 586-598). Humana Press.

Gowdhaman, D. and Ponnusami, V. 2015. Production and optimization of xylooligosaccharides from corncob by Bacillus aerophilus KGJ2 xylanase and its antioxidant potential. Int. J. Biol. Macromol.79, 595 -600.

Yang, R., Xu, S., Wang, Z. and Yang, W. 2005. Aqueous extraction of corncob xylan and production of xylooligosaccharides. LWT-Food Sci. Technol. 38(6), 677 - 682.

TAPPI. 1997. Technical association of pulp and paper industry. Atlanta, Georgia

Ai, Z., Jiang, Z., Li, L., Deng, W., Kusakabe, I. and $\mathrm{Li}, \mathrm{H}$. 2005. Immobilization
ofStreptomyces olivaceoviridis E-86 xylanase on Eudragit S-100 for xylooligosaccharide production. Process Biochem., 40(8), 2707 - 2714

Updegraff, D. 1969. Semi-micro determination of cellulose in biological material. Anal Biochem. 32, 424-428

Sluiter, A., Hames, B., Ruiz, R., Scarlata, C., Sluiter, J., Templeton, D. and Crocker, D. 2012. Determination of structural carbohydrates and lignin in biomass. National Renewable Energy Laboratory Technical Report NREL/TP-510-42618. National Renewable Energy Laboratory Golden, CO.

Selig, M., Weiss, N., Ji, Y. 2008. Enzymatic saccharification of lignocellulosic biomass- Laboratory Analytical Protocol (LAP). National Renewable Energy Laboratory Technical Report NREL/TP; 510-42629. National Renewable Energy Laboratory Golden, CO

Miller, G.L. 1959. Use of dinitrosalicylic acid reagent for determination of reducing sugar. Anal. Chem. 31(3), 426-428

Sun, Y. and Cheng, J.2002. Hydrolysis of lignocellulosic materials for ethanol production: a review. Bioresour Technol, 83(1), 1 - 11.

Tan, S.S., Li, D.Y., Jiang, Z.Q., Zhu, Y.P., Shi, B. and Li, L.T. 2008. Production of xylobiose from the autohydrolysis explosion liquor of corncob using Thermotoga maritima xylanase $\mathrm{B}$ (XynB) immobilized on nickel-chelated Eupergit C. Bioresour. Technol. 99(1), $200-204$.

Eylen, D. V., van Dongen, F., Kabel, M., de Bont, J. 2011. Corn fiber, cobs and stover: Enzyme-aided saccharification and co-fermentation after dilute acid pretreatment. Bioresour. Technol. 102(10), 5995 - 6004

Garrote, G., Domí, H. and Parajó, J.C. 2002. Autohydrolysis of corncob: study of non-isothermal operation for 
xylooligosaccharide production. J. Food Eng., 52(3), 211 - 218.

Kumar, L., Chandra, R. and Saddler, J. 2011. Influence of steam pretreatment severity on post-treatments used to enhance the enzymatic hydrolysis of pretreated softwoods at low enzyme loadings. Biotechnol. Bioeng. 108(10), 2300 2311.

Ibrahim, M.M., El-Zawawy, W.K., AbdelFattah, Y.R., Soliman, N.A. and Agblevor, F.A. 2011. Comparison of alkaline pulping with steam explosion for glucose production from rice straw. Carbohydr. Polym., 83(2), 720 - 726.

Bensah, E.C. and Mensah, M. 2013. Chemical pretreatment methods for the production of cellulosic ethanol: technologies and innovations. Int. J. Chem. Eng., 2013

Aachary, A. A., and Prapulla, S. G. 2009. Value addition to corncob: production and characterization of xylooligosaccharides from alkali pretreated lignin-saccharide complex using Aspergillus oryzae MTCC 5154. Bioresour. Technol. 100(2), 991995.

Pandiyan, K., Tiwari, R., Rana, S., Arora, A., Singh, S., Saxena, A.K. and Nain, L. 2013. Comparative efficiency of different pretreatment methods on enzymatic digestibility of Parthenium sp. World J. Microbiol. Biotechnol., 30(1), 55 - 64.

Brodeur, G., Yau, E., Badal, K., Collier, J., Ramachandran, K.B. and Ramakrishnan, S. (2011). Chemical and physicochemical pretreatment of lignocellulosic biomass: a review. Enzyme Res., 2011.

Boonchuay, P., Techapun, C., Seesuriyachan, P. and Chaiyaso, T. 2014. Production of xylooligosaccharides from corncob using a crude thermostable endoxylanase from Streptomyces thermovulgaris TISTR1948 and prebiotic properties. Food Sci. Biotechnol.23(5), 1515 - 1523

Sahare, P., Singh, R., Laxman, R.S. and Rao, M.2012. Effect of alkali pretreatment on the structural properties and enzymatic hydrolysis of corn cob. Appl. Biochem. Biotechnol., 168(7) 1806 - 1819.

Wang, Z., Keshwani, D.R., Redding, A.P. and Cheng, J.J. 2010. Sodium hydroxide pretreatment and enzymatic hydrolysis of coastal Bermuda grass. Bioresour. Technol. 101(10), 3583 - 3585

Alvira, P., Tomas-Pejo, E., Ballesteros, M. and Negro, M.J. 2010. Pretreatment technologies for an efficient bioethanol production process based on enzymatic hydrolysis: a review. Bioresour. Technol. 101(13), $4851-4861$

Kumar, P., Barrett, D.M., Delwiche, M.J., Stroeve, P. 2009. Methods for pretreatment of lignocellulosic biomass for efficient hydrolysis and biofuel production. Ind. Eng. Chem. Res. 48(8), $3713-3729$

Jain, I., Kumar, V. Satyanarayana, T. 2015. Xylooligosaccharides: an economical prebiotic from agroresidues and their health benefits. Indian J. Exp. Biol. 53, $131-142$.

\section{How to cite this article:}

Dolmani Amat and Livleen Shukla. 2021. Evaluation of Different Pretreatment Methods for Xylooligosaccharides Production. Int.J.Curr.Microbiol.App.Sci. 10(01): 517-527. doi: https://doi.org/10.20546/ijcmas.2021.1001.063 